\title{
Acoustic Emission and Shape Memory Effect in the Martensitic Transformation
}

\author{
S. Sreekala ${ }^{1}$ and G. Ananthakrishna ${ }^{1,2}$ \\ ${ }^{1}$ Materials Research Centre, Indian Institute of Science, Bangalore-560012, India \\ ${ }^{2}$ Centre for Condensed Matter Theory, Indian Institute of Science, Bangalore-560012, India
}

\begin{abstract}
Acoustic emission signals are known to exhibit a high degree of reproducibility in time and show correlations with the growth and shrinkage of martensite domains when athermal martensites are subjected to repeated thermal cycling in a restricted temperature range. We show that a recently introduced two dimensional model for the martensitic transformation mimics these features. We also show that these features are related to the shape memory effect where near full reversal of morphological features are seen under these thermal cycling conditions.
\end{abstract}

Acoustic emission (AE) is an important nondestructive technique that is sensitive to the microstructural changes taking place inside the system. It is widely employed in the detection of earthquakes of small magnitudes [1], understanding and mapping nucleation events of fracture in seismology [2], and precursor effects of fracture [3]. Its nondestructive character finds many applications in industry as well [4]. Acoustic emission is attributed to the sudden release of stored strain energy, although the details of the mechanism are generally system specific. For instance, the difference between the nature of AE signals in thermal and athermal transformations has been used to characterize the nature of a first-order transformation [5]. In the case of athermal martensites, the kinetics of the transformation is not controlled by thermal fluctuations which implies that the system is caught in a local minimum. It jumps over the barrier only when subjected to changes in external parameters such as temperature or stress [5-10]. The AE emitted during martensitic transformation often shows unusual and interesting statistical properties; one that has attracted considerable attention is the power law statistics of the AE signals $[7,9,11]$, as it is reminiscent of selforganized criticality [12]. Much less known, but perhaps equally intriguing, is that $\mathrm{AE}$ signals can show a high degree of statistical correlations in time (reproducibility) and its correlations to the growth and shrinkage of microplates $[6,8,13]$. These results based on careful experiments on single crystals of $\mathrm{CuAlZn}$ alloys under repeated thermal cycling in a restricted domain of temperatures $[8,13]$ are well summarized in Figs. 10-12 of Ref. [6]. To the best of our knowledge, these results have not been explained so far. Explaining these features is also crucial to the understanding of the shape memory effect of martensites [14]. Moreover, as far as we are aware, there are no models of shape memory effect which demonstrate the reversal of morphology of martensite domains under thermal cycling. The purpose of this paper is to devise a model which explains these correlations and provide a model for the shape memory effect.
Martensitic transformation is considered as an atypical first-order transformation as it exhibits some features of second order transition such as a precursor effect [15] and the scale free power law distribution of AE signals, again a signature of critical fluctuations. On cooling from a higher symmetry parent phase, nucleation of the thin plate like product domains with internal twinned structure is seen. Nucleation is known to be athermal usually occurring at defects [16]. Long-range strain fields resulting from the difference in the unit cell structure between the parent and product phases tend to block the transformation. The athermal nature of the transformation also means that the amount of transformed phase is fixed for a fixed quench and further undercooling is required for an additional growth. For the same reason, the transformation occurs in a broad range of temperatures and it exhibits hysteresis under thermal cycling.

The power law statistics of the avalanches has been modeled using random field Ising models [17] where the extent of quenched disorder plays a crucial role. However, as the power law is seen only after repeated thermal cycling, Vives et al. $[9,10]$ conclude that the transformation induced disorder, rather than quenched disorder (defects), is responsible for these avalanches. Recently, a model which generates dynamical disorder during the transformation has been shown to reproduce the power law statistics [11]. We show that this simple 2D phenomenological model [11] has the right ingredients to explain the correlated AE signals and the shape memory effect [14].

The model uses the deviatoric strain as the principal order parameter. The effects of the bulk and shear strains are included only in a phenomenologically way through a long-range interaction term (see below). In terms of the two components of the displacement field, $u_{x}$ and $u_{y}$, the deviatoric strain is given by $\epsilon=\left[\left(\partial u_{x}\right) /(\partial x)-\right.$ $\left.\left(\partial u_{y}\right) /(\partial y)\right] / \sqrt{2}$.

The scaled free energy, written entirely in terms of $\epsilon(\vec{r})$, is the sum of local free energy $F_{L}$ and an effective long-range term $F_{l r}$ that describes transformation 
induced strain-strain interaction. $F_{L}$ is given by

$$
F_{L}=\int d \vec{r}\left[f_{l}(\epsilon(\vec{r}))+\frac{\alpha}{2}(\vec{\nabla} \epsilon(\vec{r}))-\sigma(\vec{r}) \epsilon(\vec{r})\right],
$$

where both $\alpha$ and $\sigma$ are in scaled form. Here, $f_{l}(\epsilon(\vec{r}))$ is taken as the usual Landau polynomial for a first-order transition given by $f_{l}(\epsilon(\vec{r}))=\frac{\tau}{2} \epsilon(\vec{r})^{2}-\epsilon(\vec{r})^{4}+\frac{1}{2} \epsilon(\vec{r})^{6}$, where $\tau=\left(T-T_{c}\right) /\left(T_{0}-T_{c}\right)$ is the scaled temperature, $T_{0}$ is the first-order transition temperature, and $T_{c}$ is the temperature below which there are only two degenerate global minima $\epsilon= \pm \epsilon_{m}$. We mimic the nucleation of the martensite domains occurring at localized defect sites by an inhomogeneous stress field, $\sigma(\vec{r})$ [18]. Recent studies have shown that an effective long-range interaction between the transformed domains results due to the coupling with the other components of the strain order parameter $[15,19]$. However, as our approach is phenomenological, we attempt to guess a simple form that respects the invariance of $f_{l}(\epsilon)$ under $\epsilon \rightarrow-\epsilon$. Following Wang and Kachaturyan [20] [see their Eq. (11)], one simple form that satisfies this requirement when represented in the Fourier space can be written as

$$
F_{l r}\{\epsilon\}=\int d \vec{k} B(\vec{k} / k)\left\{\epsilon^{2}(\vec{r})\right\}_{k}\left\{\epsilon^{2}(\vec{r})\right\}_{k^{*}},
$$

where $\left\{\epsilon^{2}(\vec{r})\right\}_{k}$ and $\left\{\epsilon^{2}(\vec{r})\right\}_{k^{*}}$ is the Fourier transform of $\epsilon^{2}(\vec{r})$ and its complex conjugate, respectively, and $B(\vec{k} / k)$ is an appropriate kernel. Further, noting that the favorable directions of growth of the twins are along [11] and [1] directions, we find that the functional dependence of the kernel on $k_{x}$ and $k_{y}$ takes on a relatively simple form. We also use the fact that [10] and [01] are unfavorable directions for the growth. A simple choice of $B(\vec{k})$ having these features is $B(\vec{k} / k)=-\frac{1}{2} \beta \theta(k-\Lambda) \hat{k}_{x}^{2} \hat{k}_{y}^{2}$, where $\hat{k}_{x}$ and $\hat{k}_{y}$ are the unit vectors in the $x$ and $y$ directions, and $\beta$ is the strength of interaction. The step function $\theta(k-\Lambda)$ has been introduced to impose a cutoff on the range of the interaction. (See Ref. [21].) Equation (2) also has the property that as the transformation proceeds, the growths of the domains transverse to the [11] and [1] $]$ directions are hindered. Indeed, the geometrical picture of the kernel in real space is similar to Kartha et al. [15].

Acoustic emission during the transformation suggests that inertial effects are important [22]. This is included through the kinetic energy of the displacement fields

$$
T=\int d \vec{r} \rho\left[\left(\frac{\partial u_{x}(\vec{r}, t)}{\partial t}\right)^{2}+\left(\frac{\partial u_{y}(\vec{r}, t)}{\partial t}\right)^{2}\right],
$$

where $\rho$ is the mass density. Experiments show that the AE activity depends on the acceleration of the microplates [13]. Since the parent-product interface moves in the parent phase, it is associated with dissipation which we represent by the Rayleigh dissipative functional [23]. Again, we represent the dissipative functional entirely in terms of the deviatoric strains [22].

$$
R=\frac{1}{2} \gamma \int d \vec{r}\left(\frac{\partial}{\partial t} \epsilon(\vec{r}, t)\right)^{2} .
$$

This is consistent with the fact that shear and bulk strains are known to equilibrate rapidly and hence do not contribute. The equations of motion for the displacement fields are calculated using the Lagrangian $L=T-F$ where $F$ is the total free energy [22] through

$$
\frac{d}{d t}\left(\frac{\delta L}{\delta \dot{u}_{i}}\right)-\frac{\delta L}{\delta u_{i}}=-\frac{\delta R}{\delta \dot{u}_{i}}, \quad i=x, y .
$$

After scaling out $\rho$ and $\alpha$, the equation of motion for the deviatoric strain $\epsilon(\vec{r}, t)$ is given by

$$
\begin{aligned}
\frac{\partial^{2}}{\partial t^{2}} \epsilon(\vec{r}, t)=\nabla^{2}[ & \frac{\partial f(\vec{r}, t)}{\partial \epsilon(\vec{r}, t)}-\sigma(\vec{r})-\nabla^{2} \epsilon(\vec{r}, t)+\gamma \frac{\partial}{\partial t} \epsilon(\vec{r}, t) \\
& \left.+2 \epsilon(\vec{r}, t) \int d \vec{k} B(\vec{k} / k)\left\{\epsilon^{2}(\vec{k}, t)\right\}_{k} e^{i \vec{k} \cdot \vec{r}}\right]
\end{aligned}
$$

where $\beta$ and $\gamma$ now refer to the rescaled parameters. There are three adjustable parameters in the model, namely, the scaled temperature $\tau$, the strength of the long-range interaction $\beta$, and that of dissipation $\gamma$. The effect of increasing $\gamma$ is to reduce the twin width and that of $\beta$ is to reduce the width of the martensite domains transverse to the habit plane directions.

Simulations are carried out by discretizing Eq. (6) on a $N \times N$ grid (i.e., $\Delta x=1$ ) using the Euler's scheme with periodic boundary conditions. The time step used is $\Delta t=$ 0.002. (Actually, we see convergence of solutions even for $\Delta t=0.005$.) A pseudospectral technique is employed to compute the long-range term [11]. Computations are carried out for several values of parameters.

Our earlier study has shown that the model exhibits hysteresis under thermal cycling during which the energy dissipated $R(t),\left(=-\frac{1}{2} d E / d t\right)$ occurs in the form of bursts [11]. Using Eq. (4), we compute $R(t)$ during repeated thermal cycling in a small temperature interval as in experiments. We represent the stress field due to the random distribution of defects by $\sigma(\vec{r})=\sum_{j}^{j_{\max }} \sigma_{0}\left(\vec{r}_{j}\right) \times$ $\exp \left[-\left|\vec{r}-\vec{r}_{j}\right|^{2} / \zeta^{2}\right]$, where $\vec{r}_{j}$ refers to the coordinates of the randomly chosen defect sites and $j_{\max }$ is their total number. $\sigma_{0}\left(\vec{r}_{j}\right)$ is taken to be uniformly distributed in the interval $[-0.3,0.3]$. Using the above initial configuration, we cool the system from the austenite phase. Then, at an appropriate point [shown as in Fig. 3(b)] in the full cycle, we subject the system to repeated thermal cycling in a small temperature range $\tau_{\min }=-5$ and $\tau_{\max }=4$. The starting configuration of the martensite domains for the small cycle is chosen in such a way that it contains a fair number of martensite domains, typically, when the area fraction $\phi \approx 0.6$. For the cooling cycle, the final configuration during the heating cycle is taken as the initial configuration. Calculations are performed for a range of parameter values of $50 \leq \beta \leq 10$ and $5 \leq \gamma \leq 1$. As the general features remain the same, we report here the results for $\beta=25$ and $\gamma=4$. Other parameter values are $\Lambda=0.2, \zeta=1$, and $N=256$. During the first few cycles, the loops drift slightly in the $\phi-\tau$ plane which mimics 


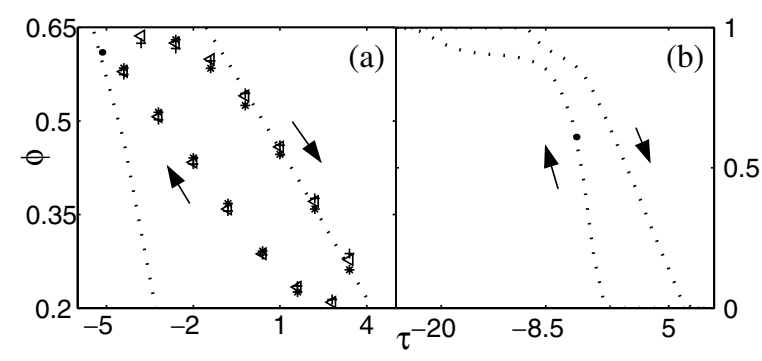

FIG. 1. (a) Area fraction $\phi$ during thermal cycling in the interval $\tau=-5$ to 4 for cycles $1(*), 2(\triangleright)$, and $3(+)$. (b) Hysteresis for the full cycle from the austenite to the martensite phase and back. (O) is the starting point of the cycles.

the training period known in experiments, eventually stabilizing after several cycles [Fig. 1(a)]. (In experiments also, the training period is typically the same for CuAlZn [6], but could be much larger in some other alloys.) A typical plot of $\phi$ vs $t$ for the first three cycles is shown in Fig. 1(a).

The energy dissipated $R(t)$ stabilizes after the training period. A plot of $R(t)$ for several forward and reverse cycles (fifth to eighth) after stabilization is shown in Fig. 2. It is clear that the energy bursts (which mimic the AE signals), as in experiments [8,13], exhibit a near repetitive pattern in time (temperature) during successive heating and cooling parts of the cycles.

In order to establish a correspondence between $R(t)$ and the changes in the spatial configuration of the martensite domains, we have simultaneously monitored the morphology over several cycles. During the first few cycles, the morphology changes considerably even as the system returns to nearly the same point in the $\phi-\tau$ diagram at the end of each cycle. Most changes occur during the first three or four cycles as can be seen from Figs. 3(a) and 3(b). Figure 3(a) is the starting morphology for the first cycle, while Fig. 3(b) is that obtained at the end of four cycles. As can be seen, all the curved twin interfaces have been rendered straight after going through four cycles. The snapshots of the morphology during one such stabilized cooling and heating cycle, the fifth one, at selected intervals are displayed in Fig. 4. It is clear that during heating, the martensite plates shrink and some even disappear. However, during cooling these domains reappear and the eventual morphology at the end of the cycle is practically recovered upon returning to the starting point on the $(\phi, \tau)$ diagram. As can be seen, the final configu-

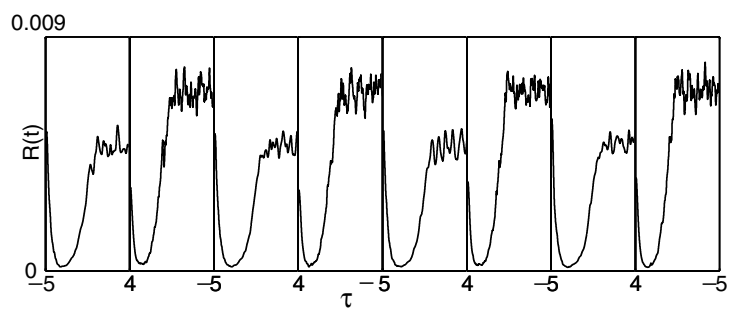

FIG. 2. Repetitive nature of $R(\tau)$ for cycles 5 to 8 . ration obtained during the fifth cycle, Fig. 4(d) can be seen to be practically the same as Fig. 3(b) which is the initial configuration for the fifth cycle. Under further cycling, the end configurations change very little, consistent with that observed in experiments [6].

Having established the repetitive nature of the energy bursts and the reversal of the morphology under thermal cycling, we need to explain the role of training cycles in inducing this correlated behavior shown in Figs. 2 and 4. We have argued earlier [11] that the model generates a huge number of transformation induced metastable states due to the fact that we have included threshold dynamics, slow driving (the rate of cooling or heating), dissipation, and a fast relaxation mechanism. The spiky nature of $R(t)$ which mimics the bursts of acoustic emission was attributed to the following. As the temperature changes, the increase in the driving force arising from the decrease in temperature goes in surmounting these local thresholds and another part goes in to the growth of the platelets, and the rest is dissipated in the form of bursts of energy (due to advancing interface). First, we note that the initial state for the repetitive small thermal cycles, Fig. 3(a), is a configuration reached by cooling from the austenite phase. Further, a comparison of Fig. 3(a) with the final morphology reached after four small thermal cycles, Fig. 3(b), reveals that the initial state Fig. 3(a) is a relatively shallow metastable state. This can be seen from the fact that the sizes of the martensitic domains of Fig. 3(a) are smaller than those in Fig. 3(b) [or Fig. 4(d)] and the twin interfaces of Fig. 3(a) are rough and considerably curved compared to Fig. 3(b). (Note that the austenite-martensite interfaces are nearly straight.) Such configurations are generally expected to have higher energy compared to straighter ones. The repetitive pattern of the energy bursts during successive cycles after the training period is an indication that the system traverses through the same set of metastable states. During the first few cycles, the free energy landscape is so modified that it smoothens out the high energy barriers corresponding to the rough curved twin interfaces in Fig. 3(a) with very little change in the area fraction. A crucial role in the smoothening process is
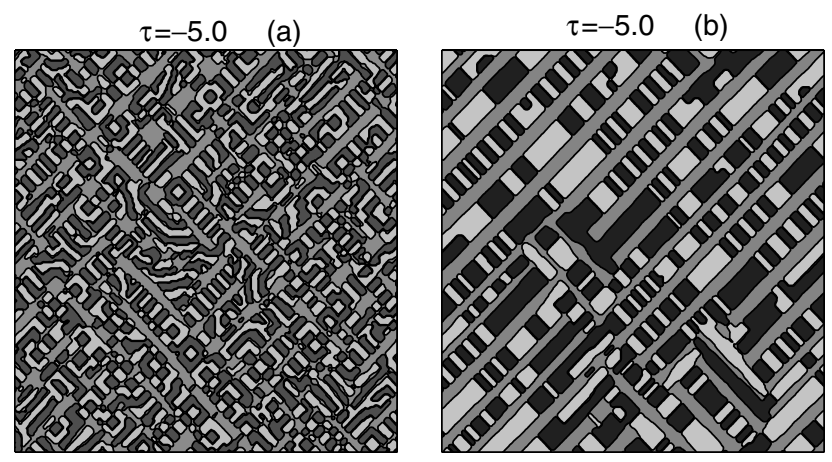

FIG. 3. Morphology of the initial configurations for (a) cycle 1, (b) cycle 4. Grey cells correspond to the austenite phase and black and white to the two martensite variants. 

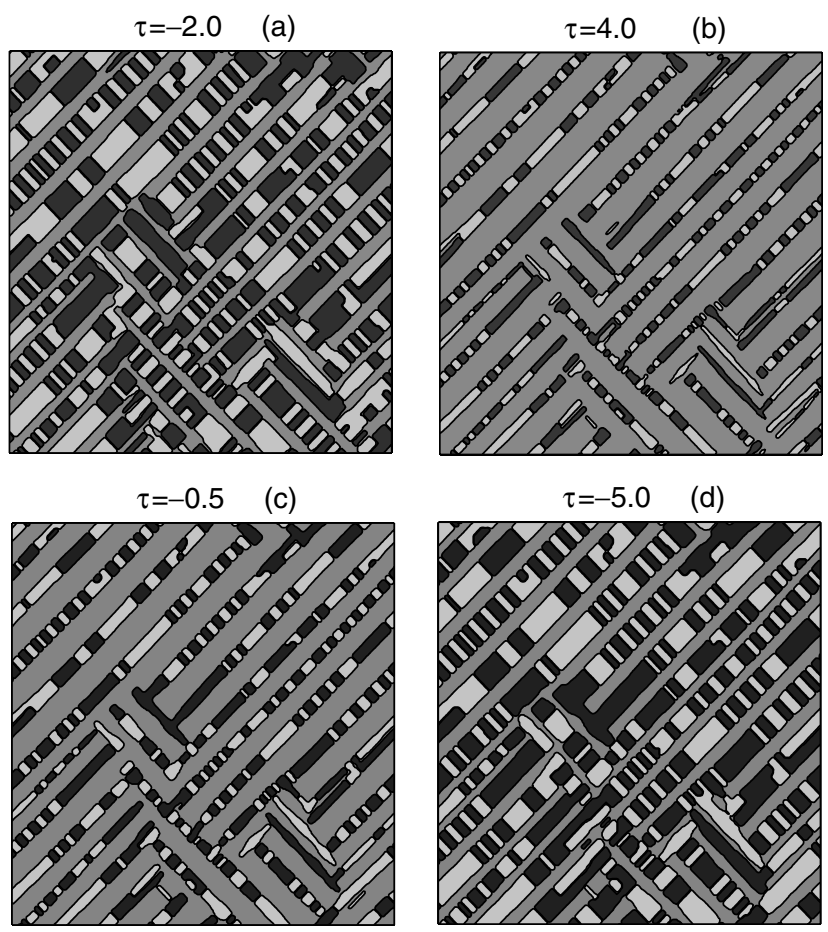

FIG. 4. Sequential morphological snapshots for $\tau=-2.0$ (a), 4.0 (b), -0.5 (c), and -5.0 (d) during the fifth cycle. The initial configuration for the cycle is shown in Fig. 3(b).

actually played by the long-range interaction term, as the growth (shrinkage) of a martensite domain is influenced by the configuration of the rest of the domains. Computation of the free energy $F_{l r}$ arising from the long-range interaction between the domains shows that it actually becomes more negative, saturating after the first few cycles. This leads to a reduction in the local free energy, $F_{L}$, as well. The net effect is to create a deeper set of metastable states for the system to circulate for the later cycles. Within one such stabilized cycle, say, the fifth, the starting configuration [Fig. 3(b)] has the lowest free energy reaching a maximum at the end of a heating cycle [Fig. 4(b)]. Thereafter it decreases during cooling. The fact that after the first few cycles, the system traverses through a deep set of free energy configurations is actually reflected in Fig. 4. Note that the morphology at $\tau=$ 4.0 is similar to that at $\tau=-0.5$, except that the widths have increased at the expense of the austenite regions consistent with the increase in the well depth stated above. The growth in the width of martensitic domains as we decrease the temperature is surprisingly similar to that observed in experiments. (See Fig. 12 of Ref.[6].)

In conclusion, our model exhibits the repetitive bursts of energy under successive thermal cycles in a small temperature interval as observed in experiments on acoustic emission. More importantly, these bursts of energy are shown to be correlated to the growth and shrinkage of martensite plates. The near full reversal of the morphology during successive cycles after the training period can be traced to the influence of the long-range interaction term. During the training period the longrange term has a tendency to smoothen out higher energy barriers in the free energy landscape. This in turn induces a transformation pathway along a unique set of low energy metastable configurations. As far as we know, this is the first time that the influence of training cycles on the shape memory effect has been elucidated. We expect that our analysis provides a good insight into the shape memory effect which finds immense applications in a variety of areas from mechanical actuators to biomedical applications [24]. To the best of the authors knowledge, this is the first model which shows near full reversal of morphology under thermal cycling.

[1] R. E. Abercombe, J. Geophys. Res. 100, No. B12, 24015 (1995).

[2] D. A. Lockner et al., Nature (London) 350, 39 (1991).

[3] A. Garcimartín et al., Phys. Rev. Lett. 79, 3202 (1997).

[4] F. J. Margetan, T. A. Gray, and R. B. Thompson, Rev. Prog. Quant. Nondestr. Eval. 10, 1721 (1991).

[5] F. J. Perez-Reche et al., Phys. Rev. Lett. 87, 195701 (2001).

[6] F. C. Lovey and V. Torra, Prog. Mater. Sci. 44, 189 (1999).

[7] Ll. Carrillo et al., Phys. Rev. Lett. 81, 1889 (1998).

[8] C. Picornell et al., Thermochim. Acta 113, 171 (1987).

[9] E. Vives et al., Phys. Rev. Lett. 72, 1694 (1994).

[10] E. Vives et al., Phys. Rev. B 52, 12644 (1995).

[11] R. Ahluwalia and G. Ananthakrishna, Phys. Rev. Lett. 86, 4076 (2001).

[12] P. Bak, C. Tang, and K. Weisenfeld, Phys. Rev. Lett., 59, 381 (1987).

[13] A. Amengual et al., Thermochim. Acta 116, 195 (1987).

[14] Note that here we are referring to a stress free situation, but the reversal of morphology under thermal cycling is basic to the usual shape memory effect induced under stress and temperature changes.

[15] S. Kartha et al., Phys. Rev. B 52, 803 (1995).

[16] A. G. Kachaturyan, Theory of Structural Transformations in Solids (Wiley, New York, 1983).

[17] J. P. Sethna et al., Phys. Rev. Lett. 70, 3347 (1993); E. Vives and A. Planes, Phys. Rev. B 50, 3839 (1994); A. Magni, Phys. Rev. B 59, 985 (1999).

[18] W. Cao, J. A. Krumhansl, and R. J. Gooding, Phys. Rev. B 41, 11319 (1990).

[19] S. R. Shenoy et al., Phys. Rev. B 60, R12 537 (1999).

[20] Y. Wang and A. G. Kachaturyan, Acta Mater. 45, 759 (1997).

[21] This choice is not unique. Further, it may be possible to have an appropriate kernel which couples bilinear terms in $\epsilon(r)$ and $\epsilon\left(r^{\prime}\right)$. However, we expect that the functional form of $B(\vec{k} / k)$ would be complicated.

[22] G. S. Bales and R. J. Gooding, Phys. Rev. Lett. 67, 3412 (1991); A. C. E. Reid and R. J. Gooding, Phys. Rev. B 50, 3588 (1994).

[23] L. D. Landau and E. M. Lifschitz, Theory of Elasticity (Pergamon, Oxford, 1986), 3rd ed.

[24] J. Van Humbeeck, M. Chandrasekaran, and L. Delaey, Endeavour, New series, 15, 148 (1991). 